\title{
BMJ Open Association of patient quality of life with the degree of agreement in the perceptions of patient disability within the stroke patient-rehabilitation therapist dyad: a cross-sectional study in postdischarge rehabilitation setting
}

\author{
Naoki Takashi (D) , ${ }^{1}$ Michael J McCarthy, ${ }^{2}$ Rie Suzuki (D) , ${ }^{3}$ Kakuya Ogahara, ${ }^{4}$ \\ Masako Ono-Kihara, ${ }^{5,6}$ Masahiro Kihara, ${ }^{5,6}$ Takeo Nakayama ${ }^{1}$
}

To cite: Takashi $\mathrm{N}$, McCarthy MJ, Suzuki R, et al. Association of patient quality of life with the degree of agreement in the perceptions of patient disability within the stroke patient-rehabilitation therapist dyad: a crosssectional study in postdischarge rehabilitation setting. BMJ Open 2021;11:e043824. doi:10.1136/ bmjopen-2020-043824

- Prepublication history and additional online supplemental material for this paper are available online. To view these files, please visit the journal online (http://dx.doi.org/10. 1136/bmjopen-2020-043824).

Received 15 August 2020

Revised 06 April 2021

Accepted 12 April 2021
Check for updates

(C) Author(s) (or their employer(s)) 2021. Re-use permitted under CC BY-NC. No commercial re-use. See rights and permissions. Published by BMJ.

For numbered affiliations see end of article.

Correspondence to Mr Naoki Takashi; 0taka8@gmail.com

\section{ABSTRACT}

Objectives The purpose of study was to explore the association between patient physical and psychological quality of life (QOL) with the degree of agreement in perceptions of patient disability within the stroke patientrehabilitation therapist dyad.

Design Cross-sectional dyadic study with a tablet-based structured questionnaire.

Setting Rehabilitation, nursing and long-term care facilities that provide rehabilitation services in the Kanagawa prefecture, Japan.

Participants The 81 dyads of a male patient with stroke living at home and the rehabilitation therapist in charge of the eligible patient were recruited from March 2019 to February 2020.

Method Patient physical and psychological QOL was measured using the WHOQOL BREF. Perceptions of patient disability were measured using the 12-item WHO Disability Assessment Schedule V.2.0 (DAS). DAS scores of patients and therapists were classified into two (high and low) and three (high, medium, low) categories, respectively, and six patterns of agreement about patient function were created and used in the analysis. Generalised estimating equations were used to examine multivariable associations between WHOQOL scores in patients and the degree of agreement within dyads adjusting for other covariates and clustering effects.

Results Among 81 enrolled dyads, 48 (59.3\%) were classified into one of four disagreement groups (low medium, low high, high medium, high low). When the patient appraised himself as having mild disability, the degree of patient-therapist disagreement was negatively associated with patient's physical and psychological QOL. When the patient appraised himself as having severe disability, his physical and/or psychological QOL was poorer, regardless of the degree of agreement.

Conclusions Disagreement in the perception of disability within patient-rehabilitation therapist dyad could be associated with patient's poor QOL, especially when the patient perceives himself as having mild disability. Reaching an agreement about patient disability is needed
Strengths and limitations of this study

- This study was the first quantitative study to evaluate the degree of agreement in a patient-therapist dyad regarding their perception of patient with stroke disability.

- The use of validated questionnaires and having one investigator conduct all of the data collection minimised measurement bias and strengthened the validity of the study.

- Multiple and diverse facilities participated in this study, which means that the findings of this study could represent the real situation of postdischarge rehabilitation services in Japan.

- The limitations of this study include the sample size and the possibility of selection bias, which may negatively affect internal validity.

- Careful consideration is required in generalising the results of this study to the entire population of patients with stroke since this study recruited only male patients with chronic phase of stroke.

in the delivery of rehabilitation care for patients with stroke living at home to improve their QOL.

\section{INTRODUCTION}

Theories on health and healing describe how patients with chronic diseases perceive their symptoms as an 'illness' in everyday life. In this regard, 'illness' represents 'human experience of symptoms and suffering.' Conversely, healthcare professionals interpret symptoms as a 'disease' based on their expert knowledge. This 'disease' stands for scientific knowledge of 'biological structure and functioning'. ${ }^{1}$ Such a difference in the perception of illness/disease between patients and healthcare professionals often 
leads to miscommunication in clinical encounters. In order to improve communication and treatment, healthcare professionals should attempt to understand patients as a whole and reach an agreement with them about the consequences of their illness and treatment goals. ${ }^{2}$ Some studies suggest that reaching an agreement in the perception of disability could lead to better outcomes, such as improved patient satisfaction with clinical communication, symptomatic control and patient mental health. ${ }^{34}$ Further, literature suggests that finding a common ground is central to delivering patient-centred care approaches. ${ }^{56}$

Reaching an agreement about the consequences of the illness and treatment goals is also vital for the delivery of effective rehabilitation services. In poststroke rehabilitation, sharing rehabilitation goals is seen as essential in the rehabilitation process because it leads to improved patient motivation and reductions in functional limitations. ${ }^{7}$ However, the majority of existing studies have been conducted in inpatient settings. ${ }^{8}$

Rosewilliam et al suggested that stroke patient-therapist communication could vary among settings where rehabilitation services are provided. ${ }^{9}$ The importance of postdischarge rehabilitation has expanded worldwide, with countries releasing guidelines advocating for continuous rehabilitation after hospital discharge. ${ }^{10-12}$ In Japan, approximately $70 \%$ of patients with stroke leave the hospital to return home, ${ }^{13}$ and these patients usually continue rehabilitation in the community under longterm care insurance (LTCI). Therefore, it is relevant to accumulate evidence regarding an agreement about the consequences of the illness and treatment goals in community-based rehabilitation services after hospital discharge. ${ }^{7}$ Three studies conducted in Japan reported that disagreement in the treatment goals commonly occur in community-based rehabilitation services, and subsequently hinder effective rehabilitation. ${ }^{14-16}$ However, the impact of such disagreement on patient outcomes is unknown.

For patients with stroke who live with disability for an extended period, disability is embedded in a personal context affected by life experiences, living environment and relationships with people who are close to them. ${ }^{17} 18$ Bendz reported that patients with stroke who live with disability for 1 year had more comprehensive perspectives about poststroke disability than their therapists. ${ }^{19}$ Consequently, during rehabilitation for patients with stroke who live in their own homes, it is even more important for rehabilitation therapists to develop a deep understanding of the patient's daily life and reach an agreement with patients regarding their perceptions of disability. ${ }^{20}$ However, no study on community-based rehabilitation has quantitatively assessed the extent of congruence in the perception of poststroke disability between therapist and patient.

This study aimed to assess the impact of agreement in the perceptions of disability on patient outcomes in postdischarge LTCI rehabilitation setting. The purpose of this study was to explore the association between the physical and psychological quality of life (QOL) in patients and the degree of agreement in the stroke patient-rehabilitation therapist dyad regarding perceptions of patient disability.

\section{METHODS}

\section{Study design}

The present study is an analytical dyadic cross-sectional study. This manuscript conforms to the Strengthening the Reporting of Observational Studies in Epidemiology criteria for reporting cross-sectional studies.

\section{Study setting}

The study was conducted at rehabilitation, nursing and long-term care facilities that provide rehabilitation services under LTCI in the Kanagawa prefecture, Japan, from 17 March 2019 to 18 February 2020. The rehabilitation services under LTCI include both outpatient and home visiting services. Outpatient services under LTCI vary, depending on the facility. Some facilities provide only physical activity during 2 hours of stay, while others offer both physical activity and recreational activities during 7 hours of stay. However, regardless of the facility, all patients receive face to face, clinic-based rehabilitation with a therapist for at least $20 \mathrm{~min}$ per day. With in-home visiting services, a therapist visits the patient's home and provides face-to-face rehabilitation for 40-60 min.

Kanagawa prefecture is one of the regional districts located in the southwest of Tokyo, which constitutes the metropolitan area. More than nine million people live in this prefecture, and the population aged 65 or more accounted for about $24.0 \%$ of the total population in 2016. The number of patients with stroke has continued to increase over the last decade in this prefecture. ${ }^{21}$

\section{Study participants}

The target population of the study was male patients with stroke who lived with their family at home and were using LTCI rehabilitation services (hereafter referred to as 'patients') as well as the rehabilitation therapists who were in charge of these patients, which included physiotherapists and occupational therapists (hereafter referred to as 'therapists'). Patients and therapists were recruited via convenience-sampling based on the eligibility criteria shown in table 1 .

The study was restricted to male patients based on the following considerations. First, we considered gender to be a factor that needs to be controlled because it affects patient-healthcare professional communication ${ }^{22}$ and predicts QOL in patients with stroke. ${ }^{23}{ }^{24}$ However, in the setting in which this study was conducted, it was challenging to recruit a sufficient number of both male and female samples that would allow us to control for gender. Because there is a significantly higher rate of stroke among men versus women who use LTCI services $(26 \%$ for males vs $12 \%$ for females ${ }^{25}$ ) in Japan, we focused on the male population in this study. 
Table 1 Eligibility criteria for male patients with stroke and rehabilitation therapists

\begin{tabular}{|c|c|c|}
\hline & Inclusion criteria & Exclusion criteria \\
\hline Patients & $\begin{array}{l}\text { 1. Diagnosis of stroke by } \\
\text { physician } \\
\text { 2. Living with family } \\
\text { caregiver } \\
\text { 3. Be able to } \\
\text { communicate and } \\
\text { give informed consent }\end{array}$ & $\begin{array}{l}\text { 1. Inability to } \\
\text { answer the } \\
\text { questionnaire (Eg } \\
\text { sensory aphasia, } \\
\text { severe cognitive } \\
\text { impairment) } \\
\text { 2. Having severe } \\
\text { comorbidities }\end{array}$ \\
\hline Therapists & $\begin{array}{l}\text { 1. Provide rehabilitation } \\
\text { for eligible patient } \\
\text { 2. Making document } \\
\text { for the patient's } \\
\text { rehabilitation plan } \\
\text { 3. Be able to } \\
\text { communicate and } \\
\text { give informed consent }\end{array}$ & \\
\hline
\end{tabular}

In this study, we originally estimated the required sample size to be 132-220, assuming 20 cases for each independent variable, ${ }^{26}$ 6-10 independent variables in the final model and a design effect of 1.1. Potential independent variables included age (years) of patients, years after stroke onset, the number of times stroke onset occurred, comorbidity (yes/no), number of years that therapists were in charge of patients, as well as the 2-6 patterns (1-5 multinomial variables) of agreement between patient and therapist in their perceptions of the patient's disability. A design effect of 1.1 was derived from the average cluster size of 2 in our pilot observation, and the maximum intraclass correlation coefficient (ICC) of a scale of QOL within the cluster by general practitioner level (ICC, 95\% CI: 0, 0-0.086) observed from the cluster randomised trials conducted among middle-aged and older adults in primary care in the previous study. ${ }^{27}$

\section{Recruitment and data collection}

Study recruitment took place in stages. First, facilities providing rehabilitation services were approached to assist with recruitment. Second, therapists in charge of eligible patients within these facilities were recruited and given leaflets to introduce the study to patients. If the patient was interested in taking part in the study, he was contacted by the first author (NT) and received an explanation about the study. The interview was conducted on the patient's preferred date.

Data collection was conducted using a tablet-based structured questionnaire. All explanations and questions were written in Japanese. Data collection for patients took place in the patient's home or in a room at the facility. Data collection for therapists took place in a room at the facility. The questionnaire was self-administered, but the first author was present during the interviews in order to monitor respondent fatigue and support respondents in using the tablet. All data collection was conducted by the first author to ensure standardised data collection, preventing interobserver differences in data collection. Key collaborators (therapists) in each facility assisted in obtaining permission and support from the facilities for sampling, recruitment and data collection. However, those therapists did not participate in data collection activities.

\section{MEASURES}

\section{Quality of life}

QOL in patients was measured using the Japanese version of the WHOQOL-BREF. ${ }^{28}{ }^{29}$ The WHOQOL-BREF is a 26-item scale administered by patients to assess subjective QOL in four domains: physical health, psychological health, social relationship and environment. Items are rated on a 5-point scale. The final scores are calculated in each domain with scores ranging from 0 to 100 , with higher scores representing better QOL. To achieve our study purpose, we used two domains as outcome variables: physical health (physical QOL) and psychological health (psychological QOL).

\section{Perceptions of patient disability in patients and therapists}

Perceptions of patient disability were measured using the Japanese version of the 12-item WHO Disability Assessment Schedule V.2.0 (DAS). ${ }^{30} 31$ This self-administered scale assesses patient disability in six main areas: (1) cognition, (2) mobility, (3) self-care, (4) getting along, (5) life activities and (6) participation. In this study, both patients and therapists reported their perceptions of the degree of difficulty the patient encountered in everyday life over the last 30 days on a scale ranging from 0 (no difficulties) to 4 (extreme difficulties/not possible at all). Patients provided ratings based on their own experiences, while therapists provided ratings based on their professional opinion.

In this study, many patients and therapists did not respond to the 12th item regarding day-to-day work because it did not apply to retired patients. Therefore, we calculated the final DAS score by summing the eleven items. Final DAS scores ranged from 0 to 44, with higher scores representing greater perceptions of patient disability.

\section{Characteristics of patients and therapists}

The following sociodemographic and disability-related characteristics were collected from patients: age, educational levels (two categories: secondary and higher education), presence of comorbidities (including hypertension, diabetes, heart diseases and cancer), recurrence of stroke (three categories: first, second and third stroke), years after stroke onset (five categories: less than 1 year, 1 year or more and less than 3years, 3 years or more and less than 5 years, 5 years or more and less than 7 years and 7 years or more) and types of rehabilitation service they used (two categories: outpatient and home visiting). Patient ages were measured continuously. As for the therapists, the following sociodemographic and occupational characteristics were collected: age (continuous), gender, occupational types (two categories: occupational therapist and physical therapist), years of 
clinical experience (five categories: 1 year or more and less than 3 years, 3 years or more and less than 5 years, 5 years or more and less than 7 years, 7 years or more and less than 10 years and 10 years or more) and years that therapist has been in charge of the patient (three categories: less than 1 year, 1year or more and less than 3years and 3years or more).

\section{Data analysis}

Descriptive statistics were used to summarise the characteristics of the patients and therapists. Mean and median values were calculated for continuous variables, while frequencies and percentages were calculated for ordinal and nominal variables.

In order to examine the degree of agreement between the patient and the therapist in their perceptions of the patient disability, we first classified patient-therapist dyads into six groups based on the patients' and therapists' DAS scores. To accomplish this classification, DAS scores from patients were dichotomised into two levels (low (L) or high $(\mathrm{H})$ ) based on the median value, while DAS scores from therapists were divided into three levels (low, medium (M) or high) based on the tertile value of patients' DAS scores. These classifications were then combined into the following six groups: DAS/LL (both DAS scores of the patient and therapist were low); DAS/LM (the patient's score was low and the therapist's score was medium); DAS/ LH (the patient's score was low and the therapist's score was high); DAS/HH (both the patient's and the therapist's scores were high); DAS/HM (the patient's score was high and the therapist's score was medium) and DAS/HL (the patient's score was high and the therapist's score was low). Both DAS/LL and DAS/HH represent 'high agreement' in DAS score between patient and therapist, DAS/LM and DAS/HM represent 'medium agreement,' and DAS/LH and DAS/HL represent 'low agreement.' Furthermore, we evaluated the distribution of differences of DAS scores between patient and therapist (patient's score minus therapist's score) to confirm the validity of categorisation, including whether there were dyads with difference of DAS scores equal to 0 , or dyads categorised into HM or HL (LM or LH), even if the patient's DAS score was lower (higher) than the therapist's score. We identified two dyads through this analysis. One dyad had difference of DAS scores of 0 . Another had difference of DAS scores of less than 0, which meant that a dyad was categorised into HM even if the patient's DAS score was lower than the therapist's score. We then evaluated the mean scores on the study outcomes, with and without these two dyads, and concluded that the values were similar (physical QOL: 44.8 vs 44.0, psychological QOL: 43.9 vs 42.3 ). Thus, these dyads were included in the study sample, and the six DAS agreement patterns were used as multinomial variables in subsequent analyses, with DAS/LL as the reference category.

Potential predictors of WHOQOL scores (physical and psychological domains) were assessed through bivariate analyses with Pearson correlation for continuous variables, Spearman rank correlation for ordinal variables, and Student's t-tests for dichotomous variables. Patient and therapist factors with $\mathrm{p}$ values less than 0.2 were adopted as covariates. ${ }^{26}$

Generalised estimating equations (GEE) with linear functions and exchangeable working correlation matrices were used to examine the multivariable association between WHOQOL scores in patients and DAS agreement patterns in dyads with covariates, adjusting for clustering effects. GEE allows exploring the association between the dependent variable and independent variables, adjusting for clustering effects, and can incorporate different numbers of observations for different clusters. ${ }^{26}$ In this study, since therapists were in charge of one to nine patients, the dyads were clustered by therapists; additionally, the number of dyads varied among clusters. Furthermore, GEE is appropriate when the study purpose is to assess the impact of the independent variables on the outcome rather than to predict the outcome of specific individuals. ${ }^{26}$ In this study, two GEE models were developed, with WHOQOL physical and psychological domain scores in patients serving as the dependent variables. Independent variables consisted of DAS agreement patterns as well as patients' and therapists' characteristics that were clinically important and scientifically meaningful or associated with patient physical or psychological QOL with a $\mathrm{p}<0.2$. The quasi-information criterion was used as an indicator of 'goodness of fit' for each model.

Moreover, we developed two additional GEE models for assessing the validity of the original model. For the first additional model, we followed the same procedure used in the original, but used a data set without the two dyads found in the evaluation of the distribution of differences between the patient's DAS score and the therapist's DAS score. We developed the second additional model using a full data set and four agreement patterns instead of six, as the main independent variable. In these four patternsDAS/LL, DAS/LH, DAS/HH, and DAS/HL-DAS scores of both the patients and therapists were divided into two categories based on the median values of the patients' scores, and then combined. While DAS/LL and DAS/HH represented agreement, DAS/LH and DAS/HL indicated disagreement.

A $p<0.05$ (two sided) was considered statistically significant. All statistical analyses were performed using SPSS V.20.0 for Windows.

\section{Ethical consideration}

This study was approved by the (Blinded for review), Ethics Committee (R1694-3), Japan. All participants provided written informed consent before participation.

\section{RESULTS}

\section{Participants}

Seventeen facilities cooperated with recruitment and data collection. Of 129 dyads across all facilities that met the eligibility criteria, 85 dyads participated in the study (participation rate was $65.9 \%$ ). The primary reason for 


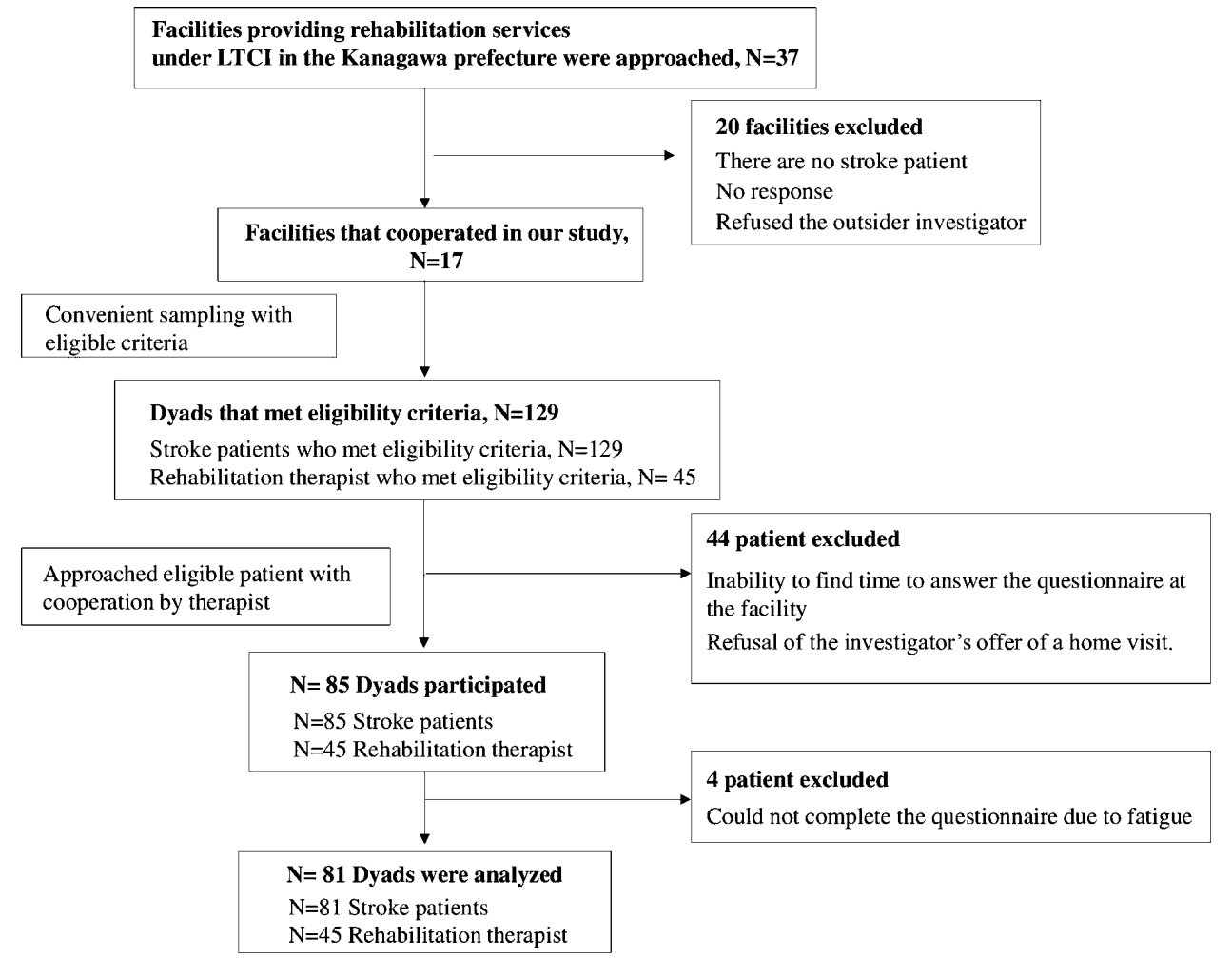

Figure 1 Participants flow chart. LTCI, long-term care insurance.

non-participation was the patients' inability to find time to answer the questionnaire at the facility coupled with their refusal of the investigator's offer of a home visit. Additionally, four dyads were excluded from the analysis because the patients could not complete the questionnaire due to fatigue. Ultimately, data from 81 dyads consisting of 81 patients and 45 therapists were analysed (figure 1 shows the participants flow chart). We did not have missing data for the sample dyads used in the analyses.

\section{Characteristics of patients and therapists}

The median age of patients was 73 years (table 2). Most were first-time patients with stroke $(74.1 \%)$, and 1 or more years had passed since the stroke onset in more than $90 \%$ of the patients. The most frequent comorbidity was hypertension, followed by diabetes. Approximately $86 \%$ of patients used outpatient service. The median age of therapists was 31 years (table 3 ), with $55.6 \%$ being male. With regard to their profession, $40 \%$ were occupational therapists and $60 \%$ were physiotherapists. More than half of the therapists had five or more years of clinical experience, and almost all had less than 3 years of a relationship with the patient of whom they were in charge.

\section{Agreement patterns of DAS scores between patients and therapists}

Table 4 details six agreement patterns of DAS scores between patients and therapists. DAS scores considerably varied between the two parties. Approximately 41\% (n=33) of dyads indicated high agreement about patient disability (DAS/LL, DAS/HH), in which the mean differences of DAS scores between patient and therapist (patient's score minus therapist's score) were 1.7 and -2.9 , respectively. Approximately 41\% (n=33) of dyads indicated medium agreement (DAS/LM, DAS/HM), in which the mean differences of DAS scores were -5.6 and 6.7 , respectively. Approximately 19\% ( $n=15)$ of dyads indicated low agreement (DAS/LH, DAS/HL), in which the mean differences of DAS scores were -17.1 and 12.3 , respectively.

\section{Bivariate association between WHOQOL scores in patients and potential predictors}

Table 5 presents the results of bivariate analyses between physical or psychological QOL in patients and potential predictor variables. Patient perceptions of their own disability were significantly negatively correlated with physical and psychological QOL ( $\mathrm{r}=-0.53, \mathrm{r}=-0.40$, respectively). Regarding DAS agreement patterns in dyads, patients' physical QOL in DAS/LH, DAS/HH, DAS/HM and DAS/ HL were significantly poor than that in DAS/LL. Additionally, patients' psychological QOL in DAS/HH, DAS/HM and DAS/HL were significantly poor than that in DAS/LL. No statistically significant associations were detected among other patient and therapist variables with patients' physical or psychological QOL.

\section{Multivariate association between patients' WHOQOL and the DAS agreement pattern in dyads with covariates}

Table 6 presents the results of the GEE analyses for the association of patients' physical and psychological QOL scores with the DAS agreement patterns between the patient and therapist. Adjusted covariates included patient comorbidities (hypertension and heart diseases) and age. We adopted the patient age as a covariate since it was considered a 
Table 2 Demographic and disability-related characteristics of male patients with stroke $(n=81)$

\begin{tabular}{|c|c|}
\hline Characteristics & $\begin{array}{l}\text { Median (IQR), mean } \\
\text { (SD), or } \mathrm{n}(\%)\end{array}$ \\
\hline \multicolumn{2}{|l|}{ Age (years) } \\
\hline Median (IQR) & $73(67.5-81.5)$ \\
\hline Mean (SD) & $72.6(11.0)$ \\
\hline \multicolumn{2}{|l|}{ Education levels: n (\%) } \\
\hline Secondary education & $40(49.4)$ \\
\hline Higher education & $41(50.6)$ \\
\hline \multicolumn{2}{|l|}{ Recurrence of stroke: $n(\%)$} \\
\hline First-time stroke & $60(74.1)$ \\
\hline Second & $14(17.3)$ \\
\hline Third or more & $7(8.6)$ \\
\hline \multicolumn{2}{|l|}{ Years after stroke onset: $n(\%)$} \\
\hline Less than 1 year & $4(4.9)$ \\
\hline 1 year or more and less than 3 years & $19(23.5)$ \\
\hline 3 years or more and less than 5 years & $14(17.3)$ \\
\hline 5 years or more and less than 7 years & $13(16.0)$ \\
\hline 7 years or more & $31(38.3)$ \\
\hline \multicolumn{2}{|l|}{ Comorbidities: n (\%) } \\
\hline Hypertension & $40(49.4)$ \\
\hline Diabetes & $21(25.9)$ \\
\hline Heart diseases & $13(16.0)$ \\
\hline Cancer & $9(11.1)$ \\
\hline Others & $12(14.8)$ \\
\hline \multicolumn{2}{|l|}{ Types of rehabilitation service: n (\%) } \\
\hline Outpatient & $70(86.4)$ \\
\hline Home-visiting & $11(13.6)$ \\
\hline \multicolumn{2}{|c|}{ Patient's score of Disability Assessment Schedule } \\
\hline Total: median (IQR) & $12(5-18)$ \\
\hline Total: mean (SD) & $12.2(7.8)$ \\
\hline \multicolumn{2}{|l|}{ WHOQOL-BREF Physical domains } \\
\hline Median (IQR) & $53.6(42.9-64.3)$ \\
\hline Mean (SD) & $53.3(16.2)$ \\
\hline \multicolumn{2}{|l|}{ WHOQOL-BREF Psychological domains } \\
\hline Median (IQR) & $50(39.6-62.5)$ \\
\hline Mean (SD) & $52.7(17.7)$ \\
\hline
\end{tabular}

DAS, Disability Assessment Schedule; WHOQOL-BREF, WHO WHOQOL-BREF quality of life assessment.

clinically important and scientifically meaningful variable, ${ }^{32}$ although in this study, it was not associated with the patient physical or psychological QOL (ie, $\mathrm{p}<0.2$ ). Consequently, our models had nine independent variables: six main predictors including six agreements patterns and three covariates (patient age, comorbidity of hypertension and comorbidity of heart disease).

In the model predicting patients' physical QOL, regression coefficients were negative and further
Table 3 Demographic and occupational characteristics of the therapists $(n=45)$

\begin{tabular}{|c|c|}
\hline Characteristics & $\begin{array}{l}\text { Median (IQR), } \\
\text { mean (SD) or } n \\
(\%)\end{array}$ \\
\hline \multicolumn{2}{|l|}{ Age (years) } \\
\hline Median (IQR) & $31(26.0-40.5)$ \\
\hline Mean (SD) & $32.7(8.1)$ \\
\hline \multicolumn{2}{|l|}{ Gender: $\mathrm{n}(\%)$} \\
\hline Male & $25(55.6)$ \\
\hline Female & $20(44.4)$ \\
\hline \multicolumn{2}{|l|}{ Occupational types: $\mathrm{n}(\%)$} \\
\hline Occupational therapists & $18(40.0)$ \\
\hline Physical therapists & $27(60.0)$ \\
\hline \multicolumn{2}{|l|}{ Years of clinical experience: $n(\%)$} \\
\hline 1 year or more and less than 3 years & $9(20.0)$ \\
\hline 3 years or more and less than 5 years & $9(20.0)$ \\
\hline 5 years or more and less than 7 years & $8(17.8)$ \\
\hline 7 years or more and less than ten years & $7(15.6)$ \\
\hline 10 years or more & $12(26.7)$ \\
\hline \multicolumn{2}{|c|}{ Years therapist has been in charge of the patient, $\mathrm{n}(\%)$} \\
\hline Less than 1 year & $41(50.6)$ \\
\hline 1 year or more and less than 3 years & $32(39.5)$ \\
\hline 3 years or more & $8(9.9)$ \\
\hline \multicolumn{2}{|c|}{ Therapist's score of Disability Assessment Schedule } \\
\hline Total: median (IQR) & $13(10-18.5)$ \\
\hline Total: mean (SD) & $14.5(7.6)$ \\
\hline
\end{tabular}

decreased as the degree of disagreement increased in the patients with low DAS scores (DAS /LL: 0 vs DAS /LM: -7.9 vs DAS /LH: -16.0$)$. There was only a statistical significance only between the patient physical QOL and DAS/LH. On the other hand, among patients with high DAS scores, the regression

Table 4 Agreement patterns of Disability Assessment Schedule (DAS) scores between patient and therapist

\begin{tabular}{|c|c|c|c|c|}
\hline \multirow[b]{2}{*}{$\begin{array}{l}\text { Agreement } \\
\text { patterns }\end{array}$} & \multirow{2}{*}{$\begin{array}{l}\text { No of } \\
\text { dyads } \\
(\%)\end{array}$} & \multirow{2}{*}{$\begin{array}{l}\text { Scores by } \\
\text { patients } \\
\text { Mean } \\
\text { (SD) }\end{array}$} & \multirow{2}{*}{$\begin{array}{l}\begin{array}{l}\text { Scores by } \\
\text { therapists }\end{array} \\
\text { Mean (SD) }\end{array}$} & \multirow{2}{*}{$\begin{array}{l}\text { Difference } \\
\text { Mean (SD) }\end{array}$} \\
\hline & & & & \\
\hline DAS/LL* & $12(14.8)$ & $5.1(2.8)$ & $3.4(2.0)$ & $1.7(3.9)$ \\
\hline DAS/LM & & $6.2(3.4)$ & $11.7(1.8)$ & $-5.6(4.0)$ \\
\hline DAS/LH & $9(11$ & $5.0(3.0)$ & $22.1(5.0)$ & $-17.1(5.2)$ \\
\hline DAS/HH & $21(25.9)$ & $19.1(6.9)$ & $22.0(4.8)$ & $-2.9(8.7)$ \\
\hline DAS/HM & 15 (18.5) & $18.0(3.9)$ & $11.3(2.6)$ & $6.7(4.6)$ \\
\hline $\mathrm{DAS} / \mathrm{HL}$ & $6(7.4)$ & 16.7 (3.2) & $4.3(2.3)$ & $12.3(3.1)$ \\
\hline
\end{tabular}

*Patient's own evaluation versus therapist's evaluation of DAS: DAS/LL=low versus low, DAS/LM=low versus medium, $\mathrm{DAS} / \mathrm{LH}=$ low versus high, DAS/HH=high versus high, DAS/ $\mathrm{HM}=$ high versus medium and $\mathrm{DAS} / \mathrm{H}=$ high versus low. 
Table 5 Bivariate association between WHOQOL scores in patients and potential predictors

\begin{tabular}{|c|c|c|c|c|c|c|}
\hline & \multicolumn{3}{|c|}{ Patients' physical QOL* } & \multicolumn{3}{|c|}{ Patients' psychological QOL† } \\
\hline & $\mathbf{r}$ & $\mathbf{t}$ & $P$ value & $\mathbf{r}$ & $\mathbf{t}$ & $P$ value \\
\hline \multicolumn{7}{|l|}{ Patients' factors } \\
\hline Higher education (ref: secondary education) & & 0.93 & 0.355 & & -0.98 & 0.331 \\
\hline Recurrence of stroke & -0.01 & & 0.927 & -0.11 & & 0.333 \\
\hline Hypertension & & 1.44 & 0.155 & & 0.09 & 0.931 \\
\hline Diabetes & & 0.03 & 0.980 & & 0.92 & 0.358 \\
\hline Heart diseases & & 1.77 & 0.081 & & 0.31 & 0.759 \\
\hline Cancer & & 0.26 & 0.796 & & 0.65 & 0.520 \\
\hline \multicolumn{7}{|l|}{ Therapists' factors } \\
\hline Age (years) & -0.01 & & 0.937 & -0.10 & & 0.356 \\
\hline Female (ref: male) & & -0.82 & 0.413 & & 0.10 & 0.919 \\
\hline Occupational therapist (ref: physical therapist) & & -0.58 & 0.566 & & -0.54 & 0.593 \\
\hline Years of clinical experience & 0.06 & & 0.577 & 0.01 & & 0.930 \\
\hline Years therapist has been in charge of the patient & -0.13 & & 0.260 & -0.08 & & 0.471 \\
\hline Therapist's score of DAS & -0.21 & & 0.064 & -0.11 & & 0.350 \\
\hline \multicolumn{7}{|c|}{ Agreement patterns of DAS between patient and therapist } \\
\hline DAS/LM $\ddagger$ (ref: DAS/LL) & & 1.50 & 0.144 & & 1.67 & 0.106 \\
\hline
\end{tabular}

*Measured by the physical domain of WHOQOL.

†Measured by the psychological domain of WHOQOL.

†Patient's own evaluation versus therapist's evaluation of DAS: DAS/LL=low versus low, DAS/LM=low versus medium, DAS/

$\mathrm{LH}=$ low versus high, $\mathrm{DAS} / \mathrm{HH}=$ high versus high, $\mathrm{DAS} / \mathrm{HM}=$ high versus medium and $\mathrm{DAS} / \mathrm{HL}=$ high versus low.

QOL, quality of life.

coefficients were negative and large (at around -20 ), regardless of the degree of agreement between patient and therapist. There were statistically significant relationships between the patient physical QOL and all three patterns (DAS/HH, DAS/HM, DAS/HL). In the model predicting patients' psychological QOL, regression coefficients of DAS/LM and DAS/LH were both negative but similar (DAS/LL: 0 vs DAS/LM: -12.4 vs DAS/LH: -10.6 ), with a statistically significant relationship detected only for DAS/LM in the patients with low DAS scores. On the other hand, in patients with high DAS scores, regression coefficients were negative and large (at around -20), regardless of the degree of agreement.

With regard to the two additional GEE models, the results of the first were similar to the original (shown in online supplemental file 1), and in the second, too, the patients' physical QOL had a similar tendency as the original. The regression coefficient of the disagreement pattern in patients with low DAS scores (DAS/ LH) was negative and larger than the agreement pattern (DAS/LL). The regression coefficient of the disagreement pattern in patients with high DAS scores (DAS/HL) was almost the same as that in the agreement pattern (DAS/HH). However, in the additional model for patients' psychological QOL, the regression coefficient in the disagreement pattern was negative and larger than in the agreement pattern, regardless of the patients' DAS scores being low or high. These results are shown in online supplemental file 2.

\section{DISCUSSION}

To our knowledge, this is the first study examining the association of patient physical and psychological QOL with the 


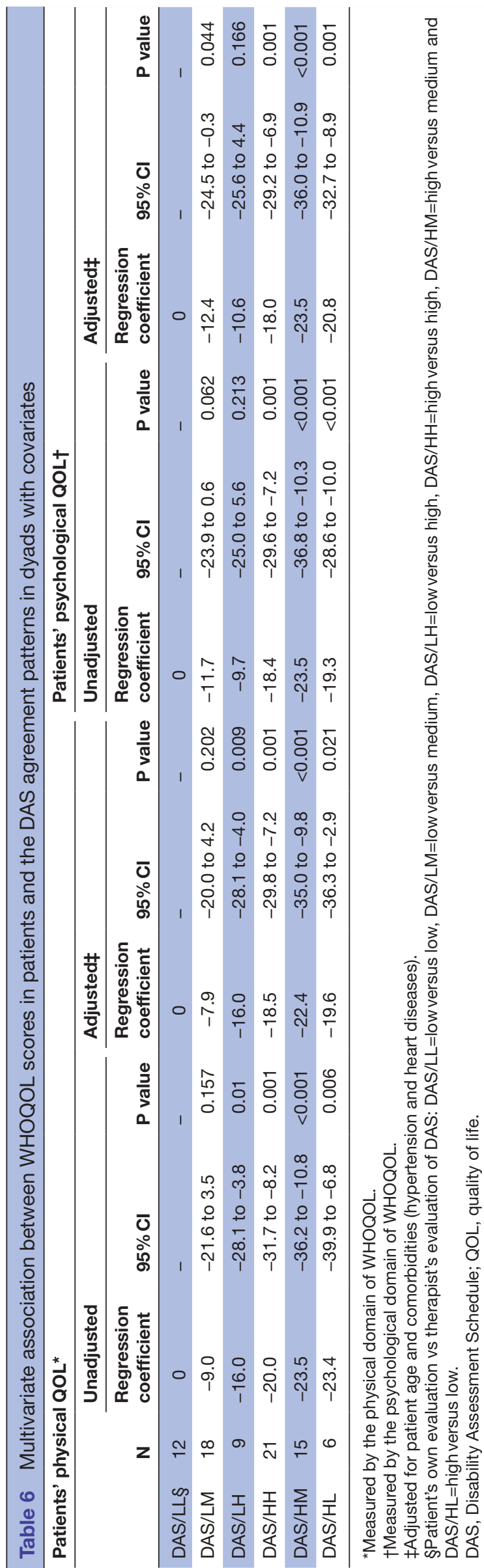

degree of agreement in the perceptions of patient disability within the stroke patient-rehabilitation therapist dyad in a Japanese postdischarge rehabilitation setting. The results indicated that $60 \%$ of dyads disagreed about the extent of the patient's disability and, notably, $20 \%$ of dyads had a high degree of disagreement. Moreover, we found that higher disagreement in the perceptions between the patient and therapist was associated with poor patient physical and psychological QOL when the patient appraised himself as having a mild disability. However, when the patient appraised himself as having a severe disability, his physical or/and psychological QOL was poor, independent of the degree of agreement.

Several studies have highlighted the disagreement in perceptions between patients with stroke and family caregivers. For example, McCarthy et al reported that disagreements in perceptions of patient function correlated with higher family caregiver depression ${ }^{33}$ and predicted higher self-rated distress in patients. ${ }^{34}$ In addition, Twiddy et al found that disagreements in the representation of illness between patients and caregivers were associated with higher distress in patients. ${ }^{35}$ The current study extended these findings by demonstrating that disagreement in the perceptions between the patient with stroke and therapist was relatively frequent and associated with poor physical and psychological QOL among patients. These results suggest that it is crucial to consider agreements in perceptions not only within patient-family caregiver dyads but also within patient-therapist dyads in order to understand the subjective physical and psychological health of a patient with stroke. In other words, the perspective of the patientfamily-therapist triad may be essential for optimal postdischarge rehabilitation.

In this study, the regression coefficients of DAS/LM and DAS/LH were negative and larger than DAS/LL in the models for patients' physical and psychological QOL. In the physical QOL model in particular, regression coefficients further decreased as the degree of disagreement increased. These results indicate that the disagreement between patient and therapist perceptions was associated with poor patient physical and psychological QOL only when the patient's own DAS score was low. This tendency was also observed in the additional model. The patient's perception of his own disability is based on various life situations, such as what activities they have participated in, what activities they have the opportunity to engage in and their living situation. ${ }^{17}{ }^{18} 36$ Furthermore, a patient's perception of disability may evolve and change over the course of recovery. ${ }^{37}$ Indeed, it is known that the patient's internal standards and values related to a subjective assessment may depend on the patient's experiences. ${ }^{38}{ }^{39}$ However, therapists generally tend to appraise the patient's disability from the perspective of impairment and functional limitation. $^{8} 940$ This professional perspective may lead therapists to overlook important points (ie, how patients with stroke have adapted to their disability) and perceive the patient's disability more pessimistically. For example, in this study, one therapist rated the disability of a patient with 
a wheelchair more severely than the patient himself. The therapist's pessimistic perception of the patient's disability may diminish the patient's confidence. Indeed, one patient who participated in our preceding exploratory qualitative study (unpublished observation) had low self-confidence and perceived that the therapist was not satisfied with his physical function. He voiced, 'Can't be helped to be underestimated because I'm sick' and 'I suffer because I don't get approved.' Thus, to improve the patient's QOL, therapists should thoroughly understand the patient from multiple perspectives, including their psychological and emotional status, the context of daily life, and adaptation of disabilities. This understanding may allow them to achieve greater agreement with the patient in perceptions of disability.

In this regard, enhancing the quality and quantity of patient-therapist communication and reaching an agreement in the perception of disability and the consequences of the illness can be critical. Previous studies on interventions have suggested that sharing and discussing the results of patient-reported outcome measures, such as QOL, between a patient and physician could enhance patient-physician communication and improve patient outcomes. ${ }^{41-43}$ Likewise, in the setting of rehabilitation for patients with stroke living at home, it can be beneficial for therapists to regularly measure the patient's perception of disability and discuss it with the patient, which may lead to greater agreement in perception and ultimately improve patient outcome.

In this study, DAS/HH, DAS/HM and DAS/HL were negatively associated with patient physical and psychological QOL. Simultaneously, regression coefficients of medium and low agreement patterns (DAS/HM, DAS/HL) were similar to the high agreement pattern (DAS/HH). This means that patients' physical and psychological QOL were generally poor, independent of the degree of agreement within the dyads when their own DAS scores were high. Although the implication of the artificial disagreement on our results may not be significant, the high agreement pattern (DAS/HH) had a sizeable standardised deviation. Therefore, there might be no notable differences in the regression coefficients among DAS/HH, DAS/HM, and DAS/HL.

Furthermore, regarding the second additional model predicting for patients' psychological QOL, the regression coefficient of the disagreement pattern (DAS/HL) was negative and larger than that of the agreement pattern (DAS/HH), in contrast to the original model. Pearce et al argued that psychological support is vital once patients face a recovery plateau, and collaborative relationships between patients and healthcare professionals, such as sharing care goals and empowering the patient, can be beneficial. ${ }^{44}$ Similarly, in this study, most patients might have been facing a recovery plateau because they lived with poststroke disability for extended periods. Therefore, the result of the second additional model may imply that the disagreements in perception within the patient-therapist dyads are likely to impact patients' psychological QOL, regardless of the degree of the patients' DAS score. Further study is needed to assess the association between disagreements in the perception of poststroke disability within patient-therapist dyads and patients' psychological QOL.

On the other hand, regarding the model predicting for patients' physical QOL, it was considered that this study's results may be robust since there was a similar tendency between the original and additional models. This means that patients' physical QOL were generally poor, independent of the degree of agreement within the dyads when patients' DAS scores were high. A previous study found that the family caregiver's optimistic perception of the patient's illness (severity of patient's fatigue) relative to the patient's own perception encouraged patients to engage in physical and social activities. ${ }^{45}$ This positive effect of disagreement in perceptions might also apply to patient-therapist relationships. A patient belonging to the DAS/HM group who participated in this study said that the rehabilitation programme gave him new experiences, such as training to use a bus, which he had never done. However, there is also a possibility that therapists who have an optimistic perception of the patient's disability miss a patient's true needs. This may lead to unmet rehabilitation needs in the patient. Unmet rehabilitation needs, in turn, are associated with unfavourable patient health outcomes. ${ }^{46}$ Considering the above, DAS/HM and DAS/HL patterns may have both positive and negative effects, which might be one of the reasons for the results of this study showing that there were no notable differences in the regression coefficients of the three patterns (DAS/HH, DAS/HM and DAS/HL).

It is known that cognitive impairment is a predictor of QOL in patients with stroke ${ }^{47}$ and a potential factor affecting patient-therapist communication. ${ }^{8}$ However, identifying cognitive decline might lead to undesirable labelling, such as stigma. ${ }^{48}$ In addition, the first author (NT) often encountered patients with stroke who felt anxious about declining cognitive function and were sensitive to receiving test results. Thus, we considered that measuring cognitive function could negatively impact the patient's mental status and did not include the cognitive test in the questionnaire. Instead, in this study, we restricted participants to patients who had no problem communicating with the therapist, and the first author, as a registered occupational therapist, observed all responding patients and confirmed that there were no problems in all responses.

This study has four limitations that must be mentioned. First, since this is a cross-sectional study, causal relationships cannot be deduced. Second, our sample size may be considered small given the number of predictor variables and covariates in the models. Indeed, the 95\% CIs of the regression coefficients in each pattern were wide. However, the sample size is in keeping with the exploratory purpose of the study and is comparable to similar dyadic studies in this population. ${ }^{359-51}$ Further research with a larger sample size, which can help obtain more accurate statistical estimates, is needed. Third, this study adopted convenience sampling with cooperation from therapists; the response rate was approximately $66 \%$. There is a possibility of selection bias because of two reasons. Therapists might feel more comfortable introducing the study to patients with whom they have a good relationship. 
Another possibility is that patients who have not had a good relationship with their therapists or have not experienced favourable rehabilitation outcomes might not be willing to take time to respond to the questionnaire. Consequently, this potential bias may lead to underestimation of the extent of disagreement in dyads. Finally, some limitations of our study were related to external validity. One or more years had passed after stroke onset in almost all patients who participated in this study, which means that they experienced daily life with poststroke disability in the community for extended periods. Experiencing various daily life events in the community may cause patients to change their perception about the consequence of illness. ${ }^{171836}$ Therefore, the degree of agreement in the perception of disability with therapists may differ between patients who live in the community after discharge and those who share a living environment in the hospital with their therapists (ie, within 6 months of stroke). Moreover, patients within the early phase after stroke onset are very likely to be in the middle of recovery, and tend to be motivated and optimistic. ${ }^{52}$ However, the patients within the chronic phase who are reaching the recovery plateau face unexpected obstacles, and therefore, the relationship with their therapist becomes more critical. ${ }^{4453}$ The impact of disagreements in perception of disability within patient-therapist dyads on patients' QOL may differ between patients in the middle of recovery and those in the plateau of recovery. Hence, caution must be exercised when generalising our findings to patients in the early phase of stroke. Another limitation is that only male patients participated in this study. Careful consideration is required to generalise the results to the population of female patients with stroke in postdischarge rehabilitation settings. Further research including female patients with stroke is highly recommended, as previous studies have shown that the QOL of patients with stroke tends to be poorer in the female population than in men. ${ }^{545}$

\section{CONCLUSION}

This study has shown that disagreements in perceptions of patient disability within patient-rehabilitation therapist dyads are negatively associated with patients' QOL, especially when the patients' perceived degrees of disability are mild. Reaching an agreement on perceptions of patient disability between the patient-therapist dyad is critical for delivering effective rehabilitation care for patients with stroke living at home and improving patient QOL.

\footnotetext{
Author affiliations

${ }^{1}$ Graduate School of Medicine, School of Public Health, Department of Health Informatics, Kyoto University, Kyoto, Japan

${ }^{2}$ College of Social and Behavioral Sciences, Department of Social Work, Northern Arizona University, Flagstaff, Arizona, USA

${ }^{3}$ Department of Public Health \& Health Sciences, University of Michigan Flint, Flint, Michigan, USA

${ }^{4}$ Department of Occupational Therapy, Kanagawa University of Human Services, Yokosuka, Japan

${ }^{5}$ Global Health Interdisciplinary Unit, Center for the Promotion of Interdisciplinary Education and Research, Kyoto University, Kyoto, Japan

${ }^{6}$ International Institute of Socio-epidemiology, Kyoto, Japan
}

Acknowledgements The authors would like to thank all patients and therapists who participated in this study. We are very grateful to key collaborators who contributed to obtain permission from the facilities and assisted first author's work related to data collection in the facility: Kanako Mizushima, OTR; Akira Maeda, RPT; Yutaka Matsui, RPT; Takahisa Uchida, OTR; Shinya Hayashi, OTR; Hiroshi Wakayama, RPT; Hideya Nagashima, RPT: Junichi Yamazaki, OTR; Yu Fudano, OTR; Tomokazu Mizuno, OTR; Keita Suzuki, OTR; Youhei Morishita, OTR; Toshizumi Sakurai, OTR. Additionally, we would like to thank Hideo Kobayashi, MD, PhD, Kounosuke Tomori, OTR, PhD (Department of occupational therapy, Tokyo University of Technology), and Machiko Oda, RPT for their special contribution to recruiting facilities.

Contributors NT is the principal author of this manuscript in all phases from the conception to the submission, data collection, data analysis and manuscript writing NT, MO-K, MK and TN conceived and designed the study. Data collection was completed by NT with support from KO. NT conducted data analysis with support from MK, MJM, RS and TN. All authors participated in interpretation of the data. All authors revised and approved the final imanuscript.

Funding The study was supported by research funding of the Department of Health Informatics, School of Public Health, Graduate School of Medicine, Kyoto University (grant number: N/A).

Competing interests None declared.

Patient consent for publication Not required.

Provenance and peer review Not commissioned; externally peer reviewed.

Data availability statement No individual participant data will be shared as participants and the facilities that cooperated in this study did not provide approval for this.

Supplemental material This content has been supplied by the author(s). It has not been vetted by BMJ Publishing Group Limited (BMJ) and may not have been peer-reviewed. Any opinions or recommendations discussed are solely those of the author(s) and are not endorsed by BMJ. BMJ disclaims all liability and responsibility arising from any reliance placed on the content. Where the content includes any translated material, BMJ does not warrant the accuracy and reliability of the translations (including but not limited to local regulations, clinical guidelines, terminology, drug names and drug dosages), and is not responsible for any error and/or omissions arising from translation and adaptation or otherwise.

Open access This is an open access article distributed in accordance with the Creative Commons Attribution Non Commercial (CC BY-NC 4.0) license, which permits others to distribute, remix, adapt, build upon this work non-commercially, and license their derivative works on different terms, provided the original work is properly cited, appropriate credit is given, any changes made indicated, and the use is non-commercial. See: http://creativecommons.org/licenses/by-nc/4.0/.

\section{ORCID iDs}

Naoki Takashi http://orcid.org/0000-0003-4360-2194

Rie Suzuki http://orcid.org/0000-0003-4969-291X

\section{REFERENCES}

1 Kleinman A. The illness narratives: suffering, healing, and the human condition (Eguchi S, Gokita S, Ueno T, trans.). New York: Basic Books, 1988.

2 Stewart M. Reflections on the doctor-patient relationship: from evidence and experience. Br J Gen Pract 2005;55:793-801.

3 Kvrgic Z, Asiedu GB, Crowson CS, et al. "Like no one is listening to me": a qualitative study of patient-provider discordance between global assessments of disease activity in rheumatoid arthritis. Arthritis Care Res 2018;70:1439-47.

4 Crespo-Lessmann A, Plaza V, González-Barcala F-J, et al. Concordance of opinions between patients and physicians and their relationship with symptomatic control and future risk in patients with moderate-severe asthma. BMJ Open Respir Res 2017;4:e000189.

5 Mead N, Bower P. Patient-centredness: a conceptual framework and review of the empirical literature. Soc Sci Med 2000;51:1087-110.

6 Constand MK, MacDermid JC, Dal Bello-Haas V, et al. Scoping review of patient-centered care approaches in healthcare. BMC Health Serv Res 2014;14:271.

7 Rose A, Rosewilliam S, Soundy A. Shared decision making within goal setting in rehabilitation settings: a systematic review. Patient Educ Couns 2017;100:65-75. 
8 Plant SE, Tyson SF, Kirk S, et al. What are the barriers and facilitators to goal-setting during rehabilitation for stroke and other acquired brain injuries? A systematic review and meta-synthesis. Clin Rehabil 2016;30:921-30.

9 Rosewilliam S, Roskell CA, Pandyan AD. A systematic review and synthesis of the quantitative and qualitative evidence behind patient-centred goal setting in stroke rehabilitation. Clin Rehabil 2011;25:501-14.

10 The Japan Stroke Society 2017. Japanese guidelines for the management of stroke 2015, 2015.

11 Winstein CJ, Stein J, Arena R. Correction to: guidelines for adult stroke rehabilitation and recovery: a guideline for healthcare professionals from the American heart Association/American stroke association. Stroke 2017;48:e78.

12 National Clinical Guideline Centre (UK). Stroke rehabilitation: long term rehabilitation after stroke. London: Royal College of Physicians, 2013.

13 Miyai I, Sonoda S, Nagai S, et al. Results of new policies for inpatient rehabilitation coverage in Japan. Neurorehabil Neural Repair 2011;25:540-7.

14 Yano $\mathrm{H}$, Yoshino T, lijima S. Examination of understanding the purpose of home-visit rehabilitation services. J Jpn Phys Ther Ass 2004;31:168-74.

15 Yoshino T, lijima S. Physical therapy for stroke outpatients: differing therapeutic aims of patients and therapists may prolong outpatient therapy. J Japanese Phys Therapy Assoc 2015;30:296-303.

16 Kamioka Y, Yoshino T, Sugaya K. Differences between stroke outpatients and physical therapists regarding the understanding of physical therapy goals. J Phys Ther Sci 2006;21:239-47.

17 Reed MC, Wood V, Harrington R, et al. Developing stroke rehabilitation and community services: a meta-synthesis of qualitative literature. Disabil Rehabil 2012;34:553-63.

18 Salter K, Hellings C, Foley N, et al. The experience of living with stroke: a qualitative meta-synthesis. J Rehabil Med 2008;40:595-602.

19 Bendz M. The first year of rehabilitation after a stroke - from two perspectives. Scand J Caring Sci 2003;17:215-22.

20 Ohura T, Tsuyama T. Reasons for starting home-visit rehabilitation services and daily life goals of users: Qualitative analysis of users' self-recorded content. Jpn Occup Ther Res 2014;33:517-25.

21 The Kanagawa Prefectural Government. Kanagawa KEN hoken iryou keikaku (in Japanses), 2020. Available: https://www.pref.kanagawa. jp/docs/t3u/cnt/f742/dainanaji.html

22 Bertakis KD. The influence of gender on the doctor-patient interaction. Patient Educ Couns 2009;76:356-60.

23 Wang Z, Li J, Wang C, et al. Gender differences in 1-year clinical characteristics and outcomes after stroke: results from the China national stroke Registry. PLoS One 2013;8:e56459.

24 Twardzik E, Clarke P, Elliott MR, et al. Neighborhood socioeconomic status and trajectories of physical health-related quality of life among stroke survivors. Stroke 2019;50:3191-7.

25 The Japanese Ministry of Health, Labor and Welfare. Comprehensive survey of living conditions (in Japanses), 2016. Available: https:// www.e-stat.go.jp/stat-search/files? page $=1$ \&layout=datalist\&toukei $=$ 00450061 \&tstat $=000001114975$ \& cycle $=7$ \&tclass $1=000001114999$ \& tclass2 $=000001115001$

26 Katz M. Multivariable analysis: a practical guide for clinicians and public health researchers. (Kihara M, Kihara-Ono M, trans.). Cambridge: Cambridge University Press, 2011.

27 Elley CR, Kerse N, Chondros P, et al. Intraclass correlation coefficients from three cluster randomised controlled trials in primary and residential health care. Aust N Z J Public Health 2005;29:461-7.

28 The WHOQOL Group. Development of the world Health organization WHOQOL-BREF quality of life assessment. Psychol Med 1988;28:551-8

29 Tazaki M, Nakane Y. A guide to WHOQOL-26. revised version. Tokyo: Kaneko Shobo, 2007.

30 Üstün TB, Kostanjsek N, Chatterji S. Measuring health and disability: manual for WHO disability assessment schedule (WHODAS 2.0). World Health Organization, 2010. https://www.who.int/classifications/ icf/whodasii/en/

31 Üstün TB, Chatterji S, Kostanjsek N. National Institutes of health developing the world Health organization disability assessment schedule 2.0. Bull World Health Organ 2010;88:815-23.

32 Paul SL, Sturm JW, Dewey HM, et al. Long-term outcome in the North East Melbourne stroke incidence study: predictors of quality of life at 5 years after stroke. Stroke 2005;36:2082-6.
33 McCarthy MJ, Lyons KS. Incongruence between stroke survivor and spouse perceptions of survivor functioning and effects on spouse mental health: a mixed-methods pilot study. Aging Ment Health 2015;19:46-54

34 McCarthy MJ, Bakas T, Schellinger J, et al. Association between incongruence about survivor function and outcomes among stroke survivors and family caregivers. Top Stroke Rehabil 2018;25:569-75.

35 Twiddy M, House A, Jones F. The association between discrepancy in illness representations on distress in stroke patients and carers. J Psychosom Res 2012;72:220-5.

36 Wasserman D, Asch A, Blustein J. Disability: definitions, models, experience. In: Zalta EN, ed. The Stanford encyclopedia of philosophy (summer 2016 edition), 2011. https://plato.stanford.edu/ archives/sum2016/entries/disability/

37 Livneh $\mathrm{H}$. The concept of time in rehabilitation and psychosocial adaptation to chronic illness and disability. Rehabil Couns Bull 2012;55:195-206.

38 Schwartz CE. Applications of response shift theory and methods to participation measurement: a brief history of a young field. Arch Phys Med Rehabil 2010;91:S38-43.

39 Daltroy LH, Larson MG, Eaton HM, et al. Discrepancies between self-reported and observed physical function in the elderly: the influence of response shift and other factors. Soc Sci Med 1999;48:1549-61.

40 Japanses Ministry of Health, Labor, and Welfare. Koureisha no chiiki ni okeru rehabilitation no aratana arikata kentoukai houkokusho (in Japanese)., 2015. Available: https://www.mhlw.go.jp/stf/shingi/otherrouken_216570.htm

41 Detmar SB, Muller MJ, Schornagel JH, et al. Health-related quality-of-life assessments and patient-physician communication: a randomized controlled trial. JAMA 2002;288:987.

42 Boyce MB, Browne JP, Greenhalgh J. The experiences of professionals with using information from patient-reported outcome measures to improve the quality of healthcare: a systematic review of qualitative research. BMJ Qual Saf 2014;23:508-18.

43 Noonan VK, Lyddiatt A, Ware P, et al. Montreal accord on patientreported outcomes (PROs) use series - Paper 3: patient-reported outcomes can facilitate shared decision-making and guide selfmanagement. J Clin Epidemiol 2017;89:125-35.

44 Pearce G, Pinnock H, Epiphaniou E, et al. Experiences of selfmanagement support following a stroke: a Meta-Review of qualitative systematic reviews. PLoS One 2015;10:e0141803.

45 Heijmans M, Ridder Dde, Bensing J. Dissimilarity in patients' and spouses' representations of chronic illness: Exploration of relations to patient adaptation. Psychol Health 1999;14:451-66.

46 Ullberg T, Zia E, Petersson J, et al. Perceived unmet rehabilitation needs 1 year after stroke: an observational study from the Swedish stroke register. Stroke 2016;47:539-41.

47 Patel MD, McKevitt C, Lawrence E, et al. Clinical determinants of long-term quality of life after stroke. Age Ageing 2007;36:316-22.

48 Garand L, Lingler JH, Conner KO, et al. Diagnostic labels, stigma, and participation in research related to dementia and mild cognitive impairment. Res Gerontol Nurs 2009;2:112-21.

49 Ekstam L, Johansson U, Guidetti S, et al. The combined perceptions of people with stroke and their carers regarding rehabilitation needs 1 year after stroke: a mixed methods study. BMJ Open 2015;5:e006784.

50 O'Connell EL, Lawson DW, New PW, et al. Agreement between patients and nurses of neurobehavioral disability following stroke in an inpatient rehabilitation setting. Disabil Rehabil 2020;42:2868-75.

51 Cameron KV, Ponsford JL, Stolwyk RJ. Do stroke survivors agree with their clinicians on the extent of their post-stroke activity limitation and participation restriction? Neuropsychol Rehabil 2020;30:1430-48.

52 Wiles R, Ashburn A, Payne S, et al. Patients' expectations of recovery following stroke: a qualitative study. Disabil Rehabil 2002;24:841-50.

53 McKevitt C, Redfern J, Mold F, et al. Qualitative studies of stroke: a systematic review. Stroke 2004;35:1499-505.

54 Reeves MJ, Bushnell CD, Howard G, et al. Sex differences in stroke: epidemiology, clinical presentation, medical care, and outcomes. Lancet Neurol 2008;7:915-26.

55 Phan HT, Blizzard CL, Reeves MJ, et al. Sex differences in long-term quality of life among survivors after stroke in the INSTRUCT. Stroke 2019;50:2299-306. 